\title{
Optimal Stopping and Constraints for Diffusion Models of Signals with Discontinuities
}

\author{
Ramūnas Girdziušas and Jorma Laaksonen \\ Helsinki University of Technology, Laboratory of Computer and \\ Information Science, P.O. Box 5400, FI-02015 HUT, Espoo, Finland \\ \{Ramunas.Girdziusas, Jorma.Laaksonen\}@hut.fi
}

\begin{abstract}
Gaussian process regression models can be utilized in recovery of discontinuous signals. Their computational complexity is linear in the number of observations if applied with the covariance functions of nonlinear diffusion. However, such processes often result in hard-tocontrol jumps of the signal value. Synthetic examples presented in this work indicate that Bayesian evidence-maximizing stopping and knowledge whether signal values are discrete help to outperform the steady state solutions of nonlinear diffusion filtering.
\end{abstract}

\section{Introduction}

Discontinuous signals can be efficiently recovered from noisy observations by employing Gaussian processes (GPs) whose mean function conditioned on the data solves nonlinear diffusion equations. Computations scale linearly with the number of data points and the increasing input dimensionality if one utilizes the additive operator splitting [12] or probabilistic simulation [3].

Nonlinear diffusion models operate upon iterative application of the principle 'smooth less where the spatial gradient of the signal is larger'. Paradoxically, such a filtering results in discontinuous jumps of signal values yielding better preserved edges and the possibility to restore signals hidden in a large-variance noise.

This work investigates nonlinear diffusion priors for Gaussian process regression. We show that diffusion can be optimally stopped long before it approaches the steady state by employing the Bayesian evidence criterion. In addition, the signal can be constrained to take either binary or any set of values in $O(n)$ number of multiplications per iteration. This is obtained by reformulating the matrix inversion of the Thomas algorithm as a dynamic programming problem.

Section 2 states Bayesian evidence-maximizing Gaussian process regression as means to solve the smoothing problem. Section 3 discusses efficient linear diffusion prior and its smoothing characteristics from the stochastic process viewpoint. Its variational interpretation and nonlinear extension is introduced in Section 4. An example of optimal stopping is given in Section 5 whereas a general approach to impose the discrete constraints on the values of the diffusion outcome is presented in Section 6. Concluding remarks are stated in Section 7. 


\section{Gaussian Process Regression}

Let us consider a grid of $n$ spatial locations and gather the observations into vector $\mathbf{y} \in \mathbb{R}^{n}$. Let us denote the model output as $\mathbf{u} \in \mathbb{R}^{n}$. If we assume that the joint predictive density of the model outcome and the hyperparameter vector $\boldsymbol{\theta}$ is unimodal, then in the absence of any specific knowledge about optimal hyperparameters $\boldsymbol{\theta}^{*}$, the location of the posterior mode can be determined by employing the Bayesian Evidence framework [7]. At the optimal point $\left(\mathbf{u}^{*}, \boldsymbol{\theta}^{*}\right)$ the following equations should hold:

$$
\begin{aligned}
& \mathbf{u}^{*}=\arg \max _{\mathbf{u}} p\left(\mathbf{y} \mid \mathbf{u}, \boldsymbol{\theta}^{*}\right) p\left(\mathbf{u} \mid \boldsymbol{\theta}^{*}\right), \\
& \boldsymbol{\theta}^{*}=\arg \max _{\boldsymbol{\theta}} p\left(\mathbf{y} \mid \mathbf{u}^{*}, \boldsymbol{\theta}\right) \frac{p\left(\mathbf{u}^{*} \mid \boldsymbol{\theta}\right)}{p\left(\mathbf{u}^{*} \mid \mathbf{y}, \boldsymbol{\theta}\right)} .
\end{aligned}
$$

Eqs. (1) and (2) indicate that the parameters and hyperparameters are solved by balancing their likelihood and prior solutions. In spite of the absence of any a priori information about the possible hyperparameter values, the Bayesian Evidence framework determines the model hyperparameters $\boldsymbol{\theta}^{*}$ that maximize the data likelihood and the ratio between the prior and posterior modes.

Let us further assume that the joint vector of the observations $\mathbf{y}$ and the model outcome $\mathbf{u}$ comes from the probability density

$$
\left(\begin{array}{l}
\mathbf{u} \\
\mathbf{y}
\end{array}\right) \sim \mathcal{N}\left(\left(\begin{array}{l}
\mathbf{0} \\
\mathbf{0}
\end{array}\right),\left(\begin{array}{lc}
\mathbf{K}_{\theta} & \mathbf{K}_{\theta} \\
\mathbf{K}_{\theta} & \mathbf{K}_{\theta}+\theta_{0} \mathbf{I}
\end{array}\right)\right) .
$$

Eq. (3) assumes that each value of the measurement vector $\mathbf{y}$ is contaminated by additive Gaussian noise, whose variance is denoted by $\theta_{0}$. The marginal distribution $p(\mathbf{u})$ represents a sample of a zero-mean Gaussian process $U$ with the covariance matrix $\mathbf{K}_{\theta} \in \mathbb{R}^{n \times n}$ which depends on a small number of hyperparameters $\boldsymbol{\theta}$ [10]. Under Eq. (3), Eqs. (1) and (2) reduce to

$$
\begin{gathered}
\mathbf{u}^{*}=\arg \min _{\mathbf{u}}\left(\frac{1}{\theta_{0}^{*}}\|\mathbf{y}-\mathbf{u}\|^{2}+\mathbf{u}^{T} \mathbf{K}_{\theta^{*}}^{-1} \mathbf{u}\right)=\left(\mathbf{I}+\theta_{0}^{*} \mathbf{K}_{\theta^{*}}^{-1}\right)^{-1} \mathbf{y}, \\
\boldsymbol{\theta}^{*}=\arg \min _{\boldsymbol{\theta}}\left(\frac{1}{\theta_{0}}\left[\left\|\mathbf{y}-\mathbf{u}^{*}\right\|^{2}+\left(\mathbf{u}^{*}\right)^{T}\left(\mathbf{y}-\mathbf{u}^{*}\right)\right]\right. \\
\left.+\ln \left[\left(2 \pi \theta_{0}\right)^{n} \operatorname{det}\left(\mathbf{K}_{\theta}+\theta_{0} \mathbf{I}\right)\right]\right) .
\end{gathered}
$$

Therefore, the GP regression minimizes the Euclidean $\|\cdot\|^{2}$ norm between the data and the model output while maintaining certain regularity properties of the model outputs via their quadratic form determined by the inverse kernel matrix $\mathbf{K}_{\theta}^{-1}$. Probabilistic Eqs. (10)-(3) complete the regression problem by providing the model selection criterion. The upper part of Eq. (5) is the best-fit likelihood $p\left(\mathbf{y} \mid \mathbf{u}^{*}, \boldsymbol{\theta}\right)$ whereas the second term represents the ratio in Eq. (2) known as the Occam's factor [7].

Whenever the true signal is discontinuous and $n>10^{3}$, well-known covariance functions such as Gaussian kernel can not be applied to construct the matrix $\mathbf{K}_{\theta}$ because Eq. (4) would blur the edges whereas Eq. (5) would be hard to interpret, evaluate and minimize. 


\section{$3 \quad$ Linear Diffusion Prior}

Consider univariate smoothing of the observations on the discrete grid $x_{0}=$ $0, x_{1}=h, x_{2}=2 h, \ldots, x_{n+1}=1$ by employing the GP model Eq. (4) with the covariance matrix whose elements $\left[\mathbf{K}_{\theta}\right]_{i j}$ are given by the function

$$
k\left(x_{i}, x_{j}\right)=\min \left[f\left(x_{i}\right), f\left(x_{j}\right)\right], \quad x \mapsto f(x)=\int_{0}^{x} g^{-1}(x) d x,
$$

where $g^{-1}(x)$ is any density function on the interval $x \in[0,1]$. In the case of $g^{-1}(x) \equiv 1$, this GP model corresponds to a priori assumption that the process $U \equiv W$ is Wiener-Levý, also known as Brownian motion (BM). It can be considered as the simplest smoothing assumption embodied in the notion of 'integrated white noise' whose paths can be simulated according to:

$$
w_{i}=w_{i-1}+\sqrt{h} z, \quad z \sim \mathcal{N}(0,1), \quad w_{0}=0 .
$$

Regression with the covariance function of Brownian motion is an important smoothing device. A great variety of GP models, including neural networks with infinite number of hidden sigmoidal or radial basis units, are unlikely to outperform this simple model. Moreover, the BM covariance matrix yields a tridiagonal inverse, which requires only $O(n)$ number of multiplications to solve Eq. (4).

Surprisingly, an extension $g^{-1}(x) \neq$ const also yields a tridiagonal inverse covariance matrix, which makes Eq. (6) applicable to spatially selective smoothing. One can derive the corresponding Ito stochastic differential equation 9]:

$$
d U_{x}=\frac{1}{2} g\left(U_{x}\right) g^{\prime}\left(U_{x}\right) d x+g\left(U_{x}\right) d W_{x}, \quad U_{0}=0 .
$$

Such an equation indicates that by postulating the model Eq. (6), we assume that the univariate data represents noisy observations of a deterministic drift perturbed with a diffusion term $g\left(U_{x}\right) d W_{x}$. GP regression with covariance as in Eq. (6) already represents a hidden diffusion. This comes in contrast to Bayesian evidence maximizing determination of the drift and diffusion terms in the case when $\theta_{0}=0$ [1].

The BM covariance function Eq. (6) can be modified for the BM process which is constrained on the other end, e.g. $u_{1}=0$ :

$$
k\left(x_{i}, x_{j}\right)=\min \left[f\left(x_{i}\right), f\left(x_{j}\right)\right]-f\left(x_{i}\right) f\left(x_{j}\right) .
$$

Such a function also yields a tridiagonal inverse covariance matrix. A very general consideration of such matrices in light of Markov processes can be found in [5].

Smoothing properties of Eqs. (6) and (9) can also be explained by a deterministic argument based on variational calculus. This view, which is discussed in the next section, is especially helpful in choosing the function $g$, clarifying the effect of the boundary conditions on the regression outcome and further extending Eqs. (6) and (9). 


\section{Nonlinear Diffusion Prior}

The presence of discontinuities demands a careful choice of the function $g(x)$, whose effect can be seen by considering the variational nature of the conditional expectation operator in the GP regression. A continuous counterpart of Eq. (4) in the case of the deformed BM covariance function, given by Eq. (66), is

$$
u^{*}=\arg \inf _{u} \int_{0}^{1}\left(\theta_{0} g(x)\left(\partial_{x} u\right)^{2}+(u-y)^{2}\right) d x, \text { s.t. } u(0)=0, \partial_{x} u(1)=0,
$$

where $y \equiv y(x)$ and $u \equiv u(x)$. In the case of the GP model Eq. (9) the last condition changes to $u(1)=0$. Boundary conditions ensure that iterative regularization produces a unique solution.

Variational calculus such as Eq. (10) is useful in deriving explicit expressions for the inverse of the covariance matrix. Summary of the results in the case of general boundary conditions can be found in Section $\mathrm{A}$.

Clearly, the function $g(x)$ acts as a spatially-dependent penalty term for the squared derivative of the model output. It can be chosen in such a way that the smoothing depends on a rough estimate of the spatial derivative of the observations [12,4]:

$$
g(x) \mapsto g[y(x)] \equiv 1-e^{-c\left(\frac{\partial_{x x \sigma}}{\lambda}\right)^{-s}} .
$$

Here the time-dependent observations $y$ are passed through a Gaussian filter of variance $\sigma^{2}$ resulting in the signal $y_{\sigma}$, whose spatial derivative's value is denoted by $\partial_{x} u_{\sigma}$. The constant $c$ can always be chosen beforehand so that the diffusion of the original noisy signal $y$ takes place only in the low derivative regions where $\left|\partial_{x} u_{\sigma}\right|<\lambda[4$. The even number $s \geq 2$ denotes the sharpness of the nonlinearity. Eq. (10) used with Eq. (11) comprises the basic step in the iterative edge-preserving filtering $[12,6$. Similar models can be found in physics, e.g. two-dimensional shear band formation in granular medium [13].

In order to reveal discontinuities, it is not sufficient to introduce Eq. (11). However, an iterative re-application of Eq. (4) with the covariance matrix defined by Eqs. (6) and (11) helps. While all the functions involved are continuous, paradoxically, the iteration eventually develops discontinuities.

If we now consider the discrete grid $x_{1}=h, x_{2}=2 h, \ldots, x_{n}=1-h$ and organize the observations into a single vector $\mathbf{u}_{0}=\mathbf{y} \in \mathbb{R}^{n}$, then the iterative application of Eq. (4) corresponds to a GP regression with the matrix $\mathbf{K}_{\theta}$ :

$$
\mathbf{I}+\theta_{0} \mathbf{K}_{\theta}^{-1}=\left(\mathbf{I}+\tau \mathbf{K}_{1}^{-1}\right)\left(\mathbf{I}+\tau \mathbf{K}_{2}^{-1}\right) \cdots\left(\mathbf{I}+\tau \mathbf{K}_{m}^{-1}\right),
$$

where the matrices $\mathbf{K}_{t}^{-1}$ are given by Eq. (17). The variance $\theta_{0}$ of a single regularization step in Eq. (10) becomes the time step size if the iterated Eq. (10) is viewed as an implicit Euler stepping of classical diffusion equations with nonlinear diffusion coefficient $g(u)$. Eq. (12) presents a way to smooth the observations in $O(n m)$ multiplications. Needless to state, the iterated composition of the inverse tridiagonal matrices comprises an efficient GP model with a non-sparse covariance matrix and its inverse. 


\section{$5 \quad$ Evidence-Maximizing Stopping}

Eq. (12), when used together with Eq. (5), gives a formal approach towards determination of numerous diffusion parameters, including a finite optimal stopping time $m$ which usually outperforms solutions that approach the steady state $m \rightarrow \infty$. In the case of a small variance $\theta_{0}$ of the additive noise, Eq. (5) reduces to 'decorrelation' between the estimated noise and the model output' 8 .

Fig. 11indicates the results obtained by filtering a synthetic signal with linear and nonlinear diffusion algorithms. The simulation was performed with $n=1000$ observations and the noise level was set to $\theta_{0}=0.44$.

Comparison of Fig. 1(a) vs. (b) and (c) vs. (d) reveals that the optimally stopped diffusion would clearly outperform the steady state in both cases. Logevidence criterion exhibits features that are similar to the mean squared error criterion between the filtering outcome and the true signal. Linear diffusion filtering blurs the edges of the signal whereas the nonlinear case preserves them.

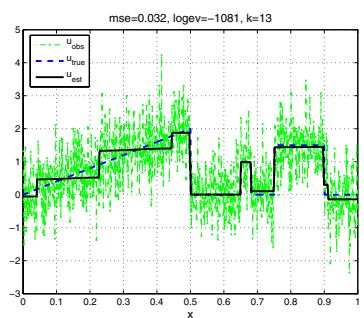

(a)

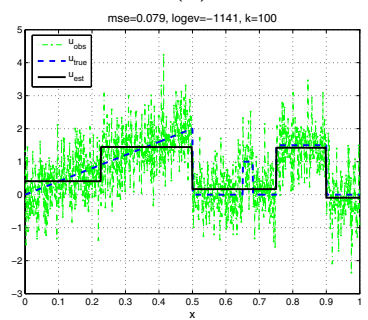

(b)

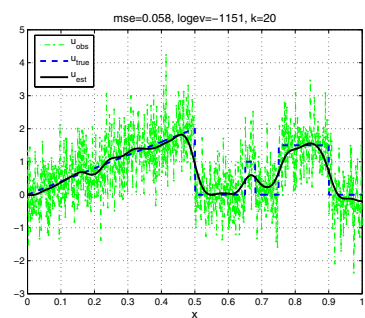

(c)

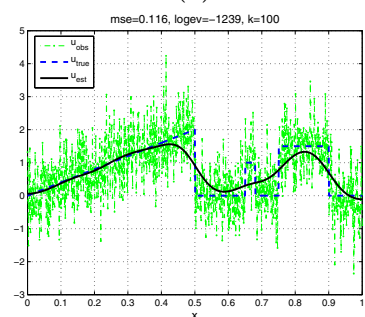

(d)

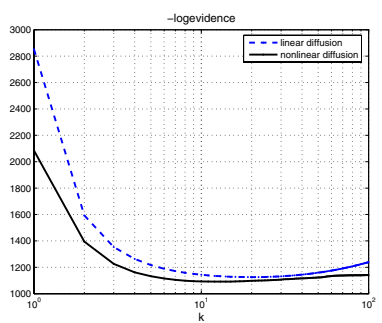

(e)

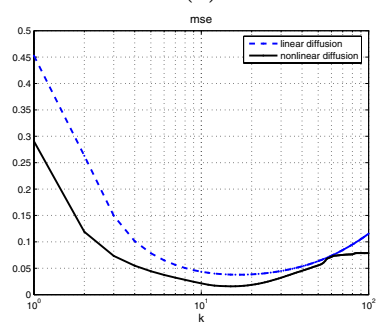

(f)

Fig. 1. Linear and nonlinear diffusion filtering from the GP regression viewpoint: (a) the outcome of nonlinear diffusion filtering at the optimal stopping time $k^{*}=13$, (b) the result approaching the steady state at $k=100$, (c) optimally stopped linear diffusion filtering at $k=20$, (d) linear diffusion at $k=100$, (e) negative logevidence as a function of the iteration counter $k$, (f) time evolution of the mean squared errors between the true and estimated signal. The stopping is performed according to negative logevidence values, which closely matches the mean squared error criterion which could be considered as a true (unknown) stopping criterion. The steady state of the linear diffusion with reflecting boundary conditions is a constant signal, i.e. the average of the observations. 


\section{Extension to Non-Gaussian Diffusion}

A computational bottleneck of the diffusion filtering lies in the evaluation of the matrix-vector product $\mathbf{u}_{t}=\left(\mathbf{I}+\tau \mathbf{K}_{t}^{-1}\right)^{-1} \mathbf{u}_{t-1}$, clf. Eq. (12). It is a common practice to implement such a procedure by applying the Thomas algorithm [12. It splits the matrix $\left(\mathbf{I}+\tau \mathbf{K}_{t}^{-1}\right)$ into a product of lower and upper-bidiagonal matrices, followed by an inversion-free forward substitution $\mathbf{L} \tilde{\mathbf{u}}_{k}=\mathbf{u}_{k-1}$ and a backward pass $\mathbf{U} \mathbf{u}_{k}=\tilde{\mathbf{u}}_{k}$. The procedure requires about $O(n)$ number of multiplications.

This computation can be viewed as a particular case of the dynamic programming approach applied to solve the variational problem in Eq. (10). Such an observation allows to introduce any discrete constraints $u \in \mathcal{U}$ into a single diffusion step with only the cost of $O(\operatorname{card}(\mathcal{U}) \cdot n)$ multiplications. In order to see this, one can define the state variable $\lambda \equiv u_{s-1}$ and introduce the value function

$$
v_{s}(\lambda)=\min _{u_{s}, \ldots, u_{n} \in \mathcal{U}} \sum_{j=s}^{n}\left[\tau g_{j}\left(u_{j}-\lambda\right)^{2}+\left(u_{j}-y_{j}\right)^{2}\right] \text {. }
$$

Solution to Eq. (10) when $u \in \mathcal{U}$ can then be solved recursively in two stages. During the backward pass, one solves $n$ one-dimensional minimization problems

$$
v_{s}(\lambda)=\min _{u_{s} \in \mathcal{U}}\left[v_{s+1}\left(u_{s}\right)+\tau g_{s}\left(u_{s}-\lambda\right)^{2}+\left(u_{s}-y_{s}\right)^{2}\right], \quad v_{n+1}=0
$$

for $s=n, n-1, \ldots, 1$ and tracks the optimal solutions $\varphi_{s}(\lambda)=\arg \min _{u_{s} \in \mathcal{U}}[\cdot]$. A solution to the constrained Eq. (10) can then be found by using the initial condition $u_{1}^{*}=0$ and tracing forward $u_{s}^{*}=\varphi_{s}\left(u_{s-1}^{*}\right), s=2, \ldots, n$. Whenever $\mathcal{U} \equiv \mathbb{R}$, an analytic solution of Eq. (14) would further reduce the computational complexity, but this formulae turns out to be just a variant of the Thomas algorithm.

The procedure based on Eqs. (13) and (14) can be derived by writing the Hamilton-Bellman-Jacobi equation for the variational problem Eq. (10) and applying the Euler algorithm to obtain its discrete counterpart 2. Alternatively, this recursive formulae can be viewed as the Viterbi algorithm used to determined the optimal state sequence of the backward hidden Markov model (HMM). Such an HMM at the location $x_{s=n}=n h$ emits the observation $y_{n}$ with the Gaussian density of mean $u_{n}$ and variance $\tau$ and jumps to the next state $s-1$ with a probability whose logarithm up to an additive constant is $\ln a_{n, s-1}=g_{n}\left(u_{n}-u_{s-1}\right)^{2}$. The case of the boundary conditions in Eq. (10) corresponds to a priori unspecified initial probability of the state $u_{s=n}$. In this manner, the process then generates all $n$ observations finally ending in the state $u_{s=1}=0$.

This generalization of the Thomas algorithm is examined in Fig. 2, A standard single step of the nonlinear diffusion performs rather poorly as in Fig. 2a, especially when the noise is non-Gaussian, clf. Fig. 2b,c. In these last two cases the nonlinear diffusion would not converge to the true signal if more iterations followed. However, if we specify the knowledge that the signal values can only become binary, the whole problem can be solved in just one iteration. 

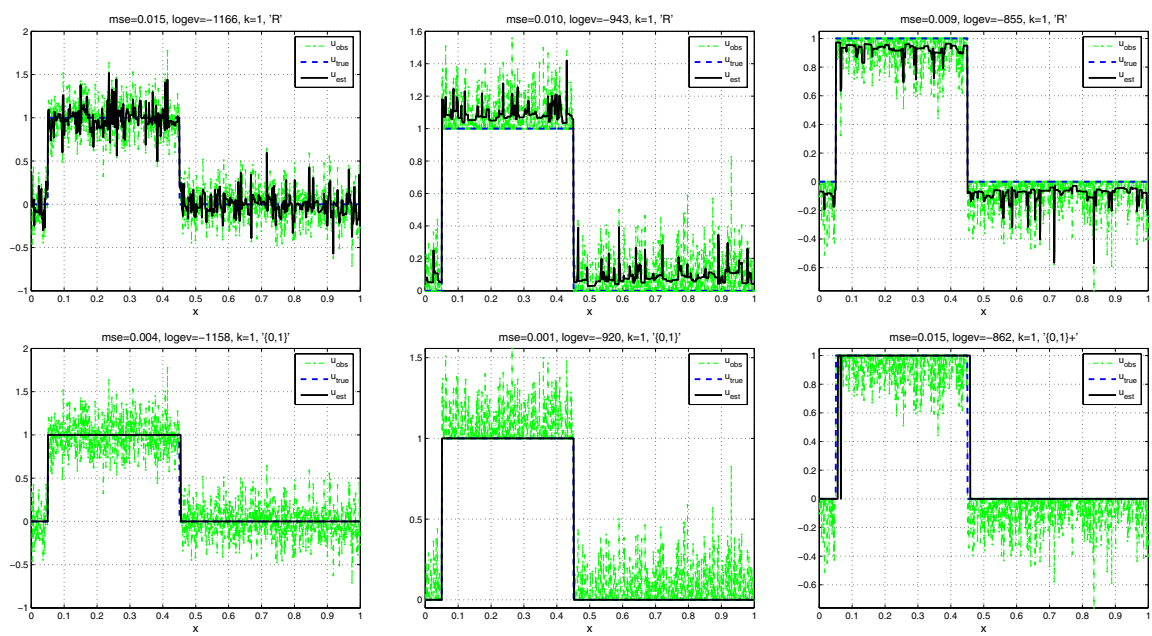

Fig. 2. Filtering a level change in additive noise. The first row illustrates the outcome of nonlinear diffusion filtering, whereas the second row shows the solutions obtained with just one iteration of diffusion whose outcome is constrained to take binary values of either 0 or 1 . The first column displays the experiment with Gaussian noise whose variance is $\theta_{0}=0.04$. The second and third columns correspond to the experiments with non-Gaussian noise, obtained by setting either positive or negative values of the noise to zero.

\section{Conclusions}

The principle of nonlinear diffusion filtering needs systematic control mechanisms to cope with discontinuities. By performing computer simulations with synthetic data, we have examined two particular solutions to such a problem: (i) optimal diffusion stopping and (ii) constraining of the diffusion outcome to take only discrete values. The first principle was implemented by viewing the diffusion filtering as Gaussian process regression. The second approach was examined by viewing the Thomas algorithm as a dynamic programming procedure. Both methods provide efficient implementations.

\section{References}

1. I. Babuška, M. Práger, E. Vitásek, and R. Radok. Numerical Processes in Differential Equations. John Wiley and Sons, Ltd., 1966.

2. R. E. Bellman and S. E. Dreyfus. Applied Dynamic Programming. Princeton University Press, 1962.

3. F.M. Buchmann and W.P. Petersen. Solving Dirichlet problems numerically using the Feynman-Kac representation. Bit Numerical Mathematics, 43(3):519-540, 2003.

4. Frederico D'Almeida. Nonlinear diffusion toolbox. MATLAB Central.

5. A. Kavčić and J.M.F. Moura. Matrices with banded inverses: inversion algorithms and factorization of Gauss-Markov processes. IEEE Trans. on Information Theory, 46(4):1495-1509, July 2000. 
6. M. Lassas and S. Siltanen. Can one use total variation prior for edge-preserving Bayesian inversion? Inverse Problems, 20:1537-1563, October 2004.

7. D.J.C. MacKay. Bayesian Interpolation. Neural Computation, 4(3):415-447, 1992.

8. P. Mrázek and M. Navara. Selection of optimal stopping time for nonlinear diffusion filtering. Int. Journal of Computer Vision, 52(2):189-203, 2003.

9. J. Nicolau. A method for simulating non-linear stochastic differential equations in $\mathbb{R}^{1}$. Technical report, Universidade Técnica de Lisboa, 2004.

10. A. O'Hagan and J. F. C. Kingman. Curve fitting and optimal design for prediction. J. R. Statist. Soc. B, 40(1):1-42, 1978.

11. N. G. Polson and G. O. Roberts. Bayes factors for discrete observations from diffusion processes. Biometrika, 8(1):11-26, March 1994.

12. J. Weickert, B. M. ter Haar Romeny, and M. A.Viergever. Efficient and reliable schemes for nonlinear diffusion filtering. IEEE Trans. on Image Processing, 7(3):398-410, March 1998.

13. T. P. Witelski. A discrete model for an ill-posed nonlinear parabolic PDE. Physica D, 160:189-221, 2001.

14. T. Yamamoto. Inversion formulas for tridiagonal matrices with applications to boundary value problems. Numer. Funct. Anal. and Optimiz., 22(4):357-385, 2001.

\section{A Inverse BM Covariance Matrix}

This summary derives the covariance matrix of GP regression with deformed BM covariance functions in the case of general boundary conditions. We use discrete approximation [1] and extend several existing results which can be found in [14.

Consider a discrete grid $x_{0}=0, x_{1}=h, x_{2}=2 h, \ldots, x_{n+1}=1$. Then a single iteration of Eq. (10) with boundary conditions

$$
\begin{aligned}
& \alpha_{1} u(0)+\beta_{1} \partial_{x} u(0)=\gamma_{1}, \\
& \alpha_{2} u(1)+\beta_{2} \partial_{x} u(1)=\gamma_{2}
\end{aligned}
$$

reduces to solving a linear system of equations, which corresponds to an optimal solution of Eq. (4) with $\mathbf{y}=\left(y_{1}-\delta_{1}, y_{2}, \cdots, y_{n-1}, y_{n}-\delta_{2}\right)^{T} \in \mathbb{R}^{n}$ and the tridiagonal inverse covariance matrix

$$
\mathbf{K}_{t}^{-1}=\frac{\tau}{h^{2}}\left(\begin{array}{ccccc}
g_{1}+g_{2} & -g_{2} & & & \\
-g_{2} & g_{2}+g_{3} & -g_{3} & & \\
& \ddots & \ddots & \ddots & \\
& & -g_{n-1} & g_{n-1}+g_{n} & -g_{n} \\
& & & -g_{n} & g_{n}+g_{n+1}
\end{array}\right)
$$

Here $\delta_{1}=g_{1} \gamma_{1} /\left(\alpha_{1}+h \beta_{1}\right), \delta_{2}=g_{n+1} \gamma_{2} /\left(\alpha_{2}+h \beta_{2}\right)$ and the elements are

$$
\begin{aligned}
g_{1} & =\left[g\left(u_{k-1}\left(x_{1}\right)\right)+g\left(u_{k-1}\left(x_{0}\right)\right)\right] h \beta_{1} /\left(\alpha_{1}+h \beta_{1}\right), \\
g_{i} & =g\left(u_{k-1}\left(x_{i}\right)\right)+g\left(u_{k-1}\left(x_{i-1}\right)\right), i=2, \ldots, n, \\
g_{n+1} & =g\left(u_{k-1}\left(x_{n+1}\right)\right)+g\left(u_{k-1}\left(x_{n}\right)\right) h \beta_{2} /\left(\alpha_{2}+h \beta_{2}\right) .
\end{aligned}
$$

The boundary conditions are preserved with $o\left(h^{2}\right)$ accuracy whereas $o\left(h^{4}\right)$ holds elsewhere. However, this is valid only if $y(x)$ is sufficiently continuous [1. In the case of a single iteration $m=1$, the constant $\tau=\theta_{0}$. When Eq. (4) is iterated, Eqs. (18)- (20) are estimated at the time instant $t-1$ according to Eq. (11) by replacing $y(x)$ with the estimate $u_{t-1}(x)$. 